\title{
The Communication of Multiple Sclerosis Diagnosis: The Patients' Perspective
}

\author{
Maria Josè Messina, Gloria Dalla Costa, Mariaemma Rodegher, Lucia Moiola, \\ Bruno Colombo, Giancarlo Comi, and Vittorio Martinelli
}

Institute of Experimental Neurology, Division of Neuroscience, Scientific Institute and University San Raffaele Hospital, Via Olgettina 48, 20132 Milan, Italy

Correspondence should be addressed to Vittorio Martinelli; martinelli.vittorio@hsr.it

Received 6 September 2015; Revised 26 November 2015; Accepted 29 November 2015

Academic Editor: Wolfgang Bruck

Copyright (C) 2015 Maria Josè Messina et al. This is an open access article distributed under the Creative Commons Attribution License, which permits unrestricted use, distribution, and reproduction in any medium, provided the original work is properly cited.

Background. Multiple sclerosis (MS) is the leading cause of nontraumatic neurological disability in young adults in Europe and in the United States. The uncertainty regarding its evolution makes the diagnosis disclosure a difficult process. Objective. The aim of the study was to provide patients' global perspective towards MS diagnosis communication. Methods. 150 consecutive patients, recently diagnosed with CIS or MS, were asked to complete a 17-item questionnaire assessing factors influencing their satisfaction with the information provided. Results. Eighty-six patients fulfilled diagnostic criteria for MS and 64 for CIS. Diagnosis disclosure took place in a private setting and required in most cases (87.3\%) less than 30 minutes. Most patients reported being moderately or highly satisfied with the information provided (75\%). The degree of satisfaction seems significantly related to patients' younger age, a longer time dedicated to disclose the diagnosis, a CIS diagnosis, and, above all, tailored information and an adequate emotional support. Conclusion. Most patients reported a good degree of satisfaction about the communication of MS or CIS diagnosis. A fruitful relationship between patient and neurologist is essential to obtain a better acceptance of the disease, patients' compliance with chronic treatments and to improve patients' quality of life.

\section{Introduction}

Multiple sclerosis (MS) is a chronic inflammatory disease of the central nervous system (CNS) and represents the leading cause of nontraumatic neurological disability in young adults in the United States and Europe. The bad common perception of MS, the uncertainty regarding the evolution of the disease, and the response to treatments make the disclosure of the diagnosis a difficult and complex process. In fact, to receive "bad news," related to an unpredictable condition, can have a big impact on the psychological well-being [1]. Since the first symptoms suggestive of MS, the disclosure of this new physical condition induces patients to a new different selfknowledge process and feelings of anxiety and depressive symptoms are frequent, especially in the first period [2]. An effective diagnosis communication is critical as it may improve acceptance of the disease, patients' compliance with chronic treatments, and, ultimately, patients' quality of life.
This is even more important considering that the revised MS diagnostic criteria allow an earlier MS diagnosis [3] and disease modifying treatments (DMTs) are now available just after the clinical onset of the disease [4]; thus more often patients start a DMT before developing a trusting and close relationship with the neurologist [5].

Despite the importance of an effective diagnosis communication, few studies on the current practice about communicating MS diagnosis have been published so far. We thus conducted a study to assess patients' opinions and perspectives regarding the manner in which physicians deliver MS diagnosis and their feedback and suggestions to improve the process.

\section{Materials and Methods}

This was a single-center, observational study conducted between July 2012 and July 2014. We included 150 consecutive 
inpatients or outpatients who received a diagnosis of clinically isolated syndrome (CIS) or MS at our or other Italian neurological departments. Eligible patients were approached by two of the authors (Vittorio Martinelli or Maria Josè Messina) who outlined the objectives and nature of the study and obtained written informed consent from willing participants. The local ethical committee approved this study as part of a large protocol on patients after the first clinical attack.

An Italian survey was developed for this study in order to assess factors affecting patients' satisfaction with the diagnosis communication. A preliminary version was assembled using the PROFILE project survey [6], which was designed to assess the factors influencing the diagnosis communication process from the Italian neurologist's perspective.

We evaluated patients' perspective using the same questionnaire referred to physicians, but with questions regarding patients' experience, to detect the different views of the two leading actors during the diagnosis communication. Two neurologists, one methodology expert, and an ad hoc focus group of 10 patients reviewed the preliminary version of the questionnaire until it had sufficient face validity to measure the concepts of interest. The final survey was a 17 -item questionnaire (see Supplementary Material available online at http://dx.doi.org/10.1155/2015/353828) collecting information on patients' demographics, timing, setting, and terminology used during the diagnosis communication. Patients were also asked about the satisfaction with the information provided, their reactions, emotional profile, and their view regarding communication issues.

Satisfaction with the information provided was recorded using a three-level scale; low, medium, or high. Univariate ordinal logistic regression was fitted between satisfaction and each of the explanatory variables to assess the crude effect of each on satisfaction. Odds ratios were estimated with the lowest category of each explanatory variable used as the reference value. A likelihood ratio (LR) test was performed to assess the overall significance of each explanatory variable. All of the explanatory variables of interest were then included in a multivariable ordinal logistic regression to estimate the adjusted odds ratios. Parameters of the ordinal logistic regression models were estimated with ridge penalization. In order to choose the optimal ridge penalization value, candidate values in the range of $0-1$ were assessed based on the resulting misclassification error. For the full model the optimal penalty used was 0.01 . All statistical analyses were performed using the computing environment $\mathrm{R}$ ( $\mathrm{R}$ Development Core Team, 2005).

\section{Results}

A total of 150 questionnaires were available for analysis. The demographic characteristics of the participants are listed in Table 1 . The median age of patients was 31 years (IQ range 2438 years). The median time since diagnosis communication was 0 days (IQ range 0-40 days). Most patients received the diagnosis in Northern Italy (81\%), and 29 (19\%) received the diagnosis in Southern or Central Italy. Eighty-six (57\%)
TABLE 1: Patients' sociodemographic and disease-related characteristics.

\begin{tabular}{lc}
\hline & Patients $(n=150)$ \\
\hline Age at onset, median (IQ range) & $30(24-38)$ \\
Gender & $101(67.3)$ \\
Females, number (\%) & $49(32.7)$ \\
$\quad$ Males, number (\%) & \\
Actual disease, ${ }^{a}$ number (\%) & $64(42.7)$ \\
$\quad$ Clinically isolated syndrome & $86(57.3)$ \\
Multiple sclerosis & $0(0.0)$ \\
Other & $121(80.7)$ \\
MS centre location, number (\%) & $7(4.7)$ \\
$\quad$ North of Italy & $22(14.7)$ \\
Centre of Italy & $1.5(1.0-2.0)$ \\
$\quad$ South of Italy & \\
EDSS at the time of diagnosis, \\
median (IQ range) \\
Time from the diagnosis, median \\
(IQ range)
\end{tabular}

${ }^{\mathrm{a}}$ Defined by the 2011 diagnostic criteria.

patients were affected by MS according to 2010 McDonald criteria and 64 (43\%) patients had a diagnosis of CIS at time of study entry. Most patients had low disability, as just 17 (11.3\%) patients had an EDSS equal to or higher than 2.0 at the time of the questionnaire. In the presence of a first neurological symptom suggestive of MS, regardless of the geographical area, most patients $(76 \%)$ had been hospitalized, while just 36 (24\%) patients had been investigated through one or more outpatient sessions. Diagnosis disclosure took place in a private setting and required in most cases $(87.3 \%)$ less than 30 minutes (it took more than 30 minutes for 14 (9.3\%) patients and more than 1 hour for only 3 (2\%) patients). Patients' family members were often present (104 patients, $69.3 \%$ ) during the communication of the diagnosis (Table 2).

\subsection{Factors Affecting Patients' Satisfaction during Diagno-} sis Disclosure. Around half of the patients reported being moderately satisfied with the information provided at the diagnosis communication (92 patients, 61.3\%) and $24(16 \%)$ patients were highly satisfied, while 34 (23.3\%) patients were not at all satisfied. The required information referred to MS diagnosis, prognosis, and available treatments.

The univariate analysis showed that most patients highly satisfied were younger, received the diagnosis in Northern Italy, and completed the questionnaire after a shorter period of time from the diagnosis $(p<0.01)$. Patients with a MS diagnosis reported significant lower satisfaction with the information provided than patients with CIS $(p<0.01)$. The setting did not seem to be as important as the spent time, as patients whose disclosure of the diagnosis took at least 30 minutes were the most satisfied. Finally, an adequate emotional support and a communication tailored to the patient's profile resulted in a higher satisfaction (Table 3). 
TABle 2: Descriptive statistics.

\begin{tabular}{|c|c|c|c|c|}
\hline \multirow{2}{*}{ Variable } & \multirow{2}{*}{ All patients } & \multicolumn{3}{|c|}{ Patient's satisfaction (\%) } \\
\hline & & Low & Medium & High \\
\hline \multicolumn{5}{|l|}{ Age at onset } \\
\hline $15-25$ & 39 & 17.9 & 56.4 & 25.6 \\
\hline $25-35$ & 61 & 21.3 & 63.9 & 14.8 \\
\hline $35-45$ & 41 & 31.7 & 56.1 & 12.2 \\
\hline $45-55$ & 9 & 11.1 & 88.9 & 0.0 \\
\hline \multicolumn{5}{|l|}{ Gender } \\
\hline Male & 49 & 26.5 & 61.2 & 12.2 \\
\hline Female & 101 & 20.8 & 61.4 & 17.8 \\
\hline \multicolumn{5}{|l|}{ Actual disease } \\
\hline Clinically isolated syndrome & 64 & 14.1 & 64.0 & 21.9 \\
\hline Multiple sclerosis & 86 & 29.1 & 59.3 & 11.6 \\
\hline \multicolumn{5}{|l|}{ Time from the diagnosis } \\
\hline Less than 30 days & 103 & 16.5 & 64.1 & 19.4 \\
\hline More than 30 days & 47 & 36.2 & 55.3 & 8.5 \\
\hline \multicolumn{5}{|l|}{ MS centre location } \\
\hline North of Italy & 121 & 18.2 & 65.3 & 16.5 \\
\hline Centre of Italy & 7 & 71.4 & 28.6 & 0.0 \\
\hline South of Italy & 22 & 31.8 & 50.0 & 18.2 \\
\hline \multicolumn{5}{|l|}{ EDSS at the time of diagnosis } \\
\hline 0 & 16 & 18.8 & 62.5 & 18.8 \\
\hline $1-2$ & 117 & 24.8 & 58.1 & 17.1 \\
\hline$>2$ & 17 & 11.8 & 82.4 & 5.9 \\
\hline \multicolumn{5}{|l|}{ Type of patient care } \\
\hline Inpatient care & 114 & 21.1 & 62.3 & 16.7 \\
\hline Outpatient care & 19 & 26.3 & 52.6 & 21.1 \\
\hline Day hospital & 17 & 29.4 & 64.7 & 5.9 \\
\hline \multicolumn{5}{|l|}{ Where did you receive the diagnosis? } \\
\hline In my hospital room & 46 & 19.6 & 63.0 & 17.4 \\
\hline In a private office & 91 & 24.2 & 58.2 & 17.6 \\
\hline In a public space & 6 & 0.0 & 100.0 & 0.0 \\
\hline Other (by phone or discharge letter) & 7 & 42.9 & 57.1 & 0.0 \\
\hline \multicolumn{5}{|c|}{ Were other people present at the time of the diagnosis? } \\
\hline No & 31 & 22.6 & 67.7 & 9.7 \\
\hline My family members & 105 & 21.9 & 59.0 & 19.0 \\
\hline Other people & 14 & 28.6 & 64.3 & 7.1 \\
\hline \multicolumn{5}{|c|}{ How long did the communication of the diagnosis take? } \\
\hline Less than 15 minutes & 67 & 35.8 & 55.2 & 9.0 \\
\hline 15-30 minutes & 65 & 15.4 & 66.2 & 18.5 \\
\hline $30-60$ minutes & 15 & 0.0 & 60.0 & 40.0 \\
\hline More than 60 minutes & 3 & 0.0 & 100 & 0.0 \\
\hline \multicolumn{5}{|c|}{ Did the neurologist provide you with the adequate emotional support? } \\
\hline No & 31 & 64.5 & 35.5 & 0.0 \\
\hline A little bit & 71 & 16.9 & 71.8 & 11.3 \\
\hline Yes & 48 & 4.2 & 62.5 & 33.3 \\
\hline \multicolumn{5}{|c|}{ Did the neurologist tailor the communication to your profile? } \\
\hline No & 38 & 50.0 & 44.7 & 5.3 \\
\hline A little bit & 82 & 14.6 & 70.7 & 14.6 \\
\hline Yes & 30 & 10.0 & 56.7 & 33.3 \\
\hline
\end{tabular}


TABLE 3: Univariate analysis of factors affecting patients' satisfaction with the disclosure of the diagnosis.

\begin{tabular}{|c|c|c|c|c|c|}
\hline Variable & OR & $\begin{array}{c}\text { Lower } \\
95 \% \text { CI }\end{array}$ & $\begin{array}{c}\text { Upper } \\
95 \% \text { CI }\end{array}$ & $p$ value & $\begin{array}{c}\text { LR } \\
p \text { value }\end{array}$ \\
\hline \multicolumn{6}{|l|}{ Age at onset } \\
\hline $15-25$ & Ref. & & & & \multirow{4}{*}{0.08} \\
\hline $25-35$ & 0.35 & 0.13 & 0.95 & 0.04 & \\
\hline $35-45$ & 0.25 & 0.08 & 0.76 & 0.01 & \\
\hline $45-55$ & 0.28 & 0.05 & 1.66 & 0.16 & \\
\hline \multicolumn{6}{|l|}{ Gender } \\
\hline Male & Ref. & & & & \\
\hline Female & 1.61 & 0.68 & 3.81 & 0.28 & 0.24 \\
\hline \multicolumn{6}{|l|}{ Actual disease } \\
\hline Clinically isolated syndrome & Ref. & & & & \\
\hline Multiple sclerosis & 0.57 & 0.24 & 1.36 & 0.21 & 0.24 \\
\hline \multicolumn{6}{|l|}{ Time from the diagnosis } \\
\hline Less than 30 days & Ref. & & & & \\
\hline More than 30 days & 1.06 & 0.35 & 3.20 & 0.92 & 0.89 \\
\hline \multicolumn{6}{|l|}{ MS centre location } \\
\hline North of Italy & Ref. & & & & \multirow{3}{*}{0.17} \\
\hline Centre of Italy & 0.14 & 0.01 & 1.59 & 0.11 & \\
\hline South of Italy & 1.26 & 0.36 & 4.48 & 0.72 & \\
\hline \multicolumn{6}{|l|}{ EDSS at the time of diagnosis } \\
\hline 0 & Ref. & & & & \multirow{3}{*}{0.93} \\
\hline $1-2$ & 1.10 & 0.30 & 3.92 & 0.88 & \\
\hline$>2$ & 0.87 & 0.17 & 4.49 & 0.87 & \\
\hline \multicolumn{6}{|l|}{ Type of patient care } \\
\hline Inpatient care & Ref. & & & & \multirow{3}{*}{0.38} \\
\hline Outpatient care & 1.70 & 0.50 & 5.78 & 0.39 & \\
\hline Day hospital & 0.54 & 0.14 & 2.07 & 0.37 & \\
\hline \multicolumn{6}{|l|}{ Where did you receive the diagnosis? } \\
\hline In my hospital room & Ref. & & & & \multirow{4}{*}{0.55} \\
\hline In a private office & 1.06 & 0.40 & 2.85 & 0.90 & \\
\hline In a public space & 0.35 & 0.05 & 2.60 & 0.31 & \\
\hline Other (by phone or discharge letter) & 0.33 & 0.05 & 2.38 & 0.27 & \\
\hline \multicolumn{6}{|c|}{ Were other people present at the time of the diagnosis? } \\
\hline No & Ref. & & & & \multirow{3}{*}{0.50} \\
\hline My family members & 0.64 & 0.23 & 1.77 & 0.39 & \\
\hline Other people & 0.37 & 0.07 & 1.83 & 0.22 & \\
\hline \multicolumn{6}{|c|}{ How long did the communication of the diagnosis take? } \\
\hline Less than 15 minutes & Ref. & & & & \multirow{4}{*}{0.15} \\
\hline 15-30 minutes & 2.19 & 0.91 & 5.27 & 0.08 & \\
\hline $30-60$ minutes & 4.67 & 1.00 & 21.87 & 0.05 & \\
\hline More than 60 minutes & 3.14 & 0.19 & 51.28 & 0.42 & \\
\hline \multicolumn{6}{|c|}{ Did the neurologist provide you with the adequate emotional support? } \\
\hline No & Ref. & & & & \multirow{3}{*}{$<0.001$} \\
\hline A little bit & 4.96 & 1.54 & 15.96 & $<0.01$ & \\
\hline Yes & 17.72 & 4.53 & 69.38 & $<0.0001$ & \\
\hline \multicolumn{6}{|c|}{ Did the neurologist tailor the communication to your profile? } \\
\hline No & Ref. & & & & \multirow{3}{*}{0.08} \\
\hline A little bit & 2.68 & 0.96 & 7.49 & 0.06 & \\
\hline Yes & 4.36 & 1.15 & 16.45 & 0.03 & \\
\hline
\end{tabular}


The multivariate ordinal logistic regression model confirmed these results, as most patients highly satisfied with the information provided were younger $(p=0.04)$, received a diagnosis of CIS $(p<0.001)$, experienced an adequate emotional support $(p<0.001)$, and reported a communication tailored to the patient's profile $(p=0.058)$ (Table 4$)$.

\subsection{Patients' Feedback and Suggestions to Improve the Diagno-} sis Communication Process. Concerning timing of diagnosis communication, the vast majority of patients (90\%) preferred to receive the communication during the diagnostic workup, whereas only $10 \%$ chose to postpone the communication after the second neurological episode. In the diagnosis communication, the presence of an expert psychologist in a MS center is perceived as relevant in changing the management of emotionally difficult situations such as the moment of communicating the diagnosis. At the same time, $67.3 \%$ of patients found it to be useful to take part in public meeting with other patients sharing their experiences and perspectives. Furthermore, $51.3 \%$ of patients believed that an additional information aid could be useful to effectively contribute to improving patients' knowledge about the disease and the available treatments.

\section{Discussion}

The reaction to the diagnosis of a chronic, disabling disease with an unpredictable course such as MS can be very destructive [6-8]. Given this, many neurologists still hesitate to pursue a MS diagnosis since the first symptoms or often use nonspecific terms such as "inflammatory disease" or "multifocal demyelinating disease of the CNS," as in our study that emerged. Though, a prompt and effective communication of the diagnosis seems to be critical for improving patients' acceptance of the disease and compliance [9-12]. In our study, the vast majority of patients (90\%) preferred to receive the communication during the diagnostic workup, whereas only $10 \%$ would have chosen to postpone the communication after the second neurological episode. These results confirm the findings of previous studies. In a Greek survey [13, 14] assessing MS patients' opinions about their experience in receiving the diagnosis, $91 \%$ of patients referred to the fact that they would prefer to know immediately the diagnosis, but only $44 \%$ actually experienced this condition. According to $76.8 \%$ of patients, a delay in the disclosure of the diagnosis would decrease their trust in their neurologist. In another study, Brown et al. [15] found that patients who were not adequately informed and could not actively participate in the management of the disease presented an increased risk of depression.

In our study, around $75 \%$ of the patients reported being moderately or highly satisfied with the information provided and with the whole communication process they had experienced. The degree of satisfaction was significantly related to a longer time dedicated to the process, except for patients who required more than 60 minutes to be informed, for whom a high level of anxiety, denial towards the disease, or other rare psychological conditions may be supposed. In our study, the setting (place where the diagnosis was disclosed and presence or not of patients' relatives) was not as important as the time devoted to the diagnosis communication for patients' satisfaction. Though, the most important factors influencing patients' satisfaction with the diagnosis were tailored information provision and, above all, an adequate emotional support. These data pieces confirm previously published findings on the importance of emotional support in the disclosure of a MS diagnosis [16]. Indeed, there is a longstanding tension in the physician's role since the twentieth century, with doctors striving for detachment to reliably care for all patients regardless of their personal feelings. Though, clinical empathy has been proven to be a powerful tool that health professionals can use to deliver care that is adapted to patients' emotional, cognitive, and biological needs. Clinical empathy involves the ability to understand the patient's inner experiences and perspective and a capability to communicate this understanding. This ultimately enables strengthening the therapeutic relationship and the patient's trust in the neurologist.

Our results confirm that a dedicated individual approach could help to ensure that patients assimilated and did not misunderstand the information provided, as previously found by Heesen et al. [17]. Nevertheless, patients' emotional state at the moment of the diagnosis makes it harder to understand the information provided, and questions or doubts could arise after the initial communication of the diagnosis. In fact about $50 \%$ of our patients reported an information aid could be useful to effectively improve their knowledge on the disease and the available treatments even further. It is well known that many patients, after the communication of the diagnosis, often seek more information on the Internet, so the quality of their knowledge is often misleading or inadequate. Therefore an information aid specifically prepared for newly diagnosed patients could provide the patient with good-quality medical information accessible anytime and anywhere. In a recent Italian multicenter study [18, 19], the effectiveness of an information aid for newly diagnosed MS patients was assessed. Patients who received the material during the trial showed a higher satisfaction with care, compared to patients without information aid. The content was considered safe and anxiety did not increase in the intervention group.

Finally, in our study most patients (67\%) suggested public meetings with other patients, sharing directly their experiences and perspectives, could be useful to improve their coping strategies after the communication of the diagnosis. In fact, other patients who have experienced the same situation could have a better understanding of the questions and doubts patients face after the initial diagnosis and could help solve them and reach a better understanding of the medical information provided.

The relatively small sample size and the prevalent involvement of patients from the Northern Italian MS centers may be some limitations of our study, as whether the sample is really representative of Italian MS patients could be questioned. Also, the type of symptoms occurred and the presence of anxiety and depression at baseline was not assessed. These may have an influence on our results, especially considering 
TABLE 4: Multivariate analysis of factors affecting patients' satisfaction with the disclosure of the diagnosis.

\begin{tabular}{|c|c|c|c|c|c|}
\hline Variable & OR & $\begin{array}{c}\text { Lower } \\
95 \% \text { CI }\end{array}$ & $\begin{array}{c}\text { Upper } \\
95 \% \text { CI }\end{array}$ & $p$ value & $\begin{array}{c}\text { LR } \\
p \text { value }\end{array}$ \\
\hline \multicolumn{6}{|l|}{ Age at onset } \\
\hline $15-25$ & Ref. & & & & \multirow{4}{*}{0.26} \\
\hline $25-35$ & 0.65 & 0.30 & 1.43 & 0.28 & \\
\hline $35-45$ & 0.42 & 0.18 & 1.00 & 0.05 & \\
\hline $45-55$ & 0.61 & 0.15 & 2.48 & 0.48 & \\
\hline \multicolumn{6}{|l|}{ Gender } \\
\hline Male & Ref. & & & & \\
\hline Female & 1.43 & 0.73 & 2.81 & 0.30 & 0.30 \\
\hline \multicolumn{6}{|l|}{ Actual disease } \\
\hline Clinically isolated syndrome & Ref. & & & & \\
\hline Multiple sclerosis & 0.44 & 0.22 & 0.85 & 0.01 & 0.01 \\
\hline \multicolumn{6}{|l|}{ Time from the diagnosis } \\
\hline Less than 30 days & Ref. & & & & \\
\hline More than 30 days & 0.36 & 0.18 & 0.74 & $<0.01$ & $<0.01$ \\
\hline \multicolumn{6}{|l|}{ MS centre location } \\
\hline North of Italy & Ref. & & & & \multirow{3}{*}{$<0.01$} \\
\hline Centre of Italy & 0.10 & 0.02 & 0.53 & $<0.01$ & \\
\hline South of Italy & 0.67 & 0.27 & 1.66 & 0.39 & \\
\hline \multicolumn{6}{|l|}{ EDSS at the time of diagnosis } \\
\hline 0 & Ref. & & & & \multirow{3}{*}{0.89} \\
\hline $1-2$ & 0.79 & 0.29 & 2.13 & 0.64 & \\
\hline$>2$ & 0.86 & 0.23 & 3.17 & 0.82 & \\
\hline \multicolumn{6}{|l|}{ Type of patient care } \\
\hline Inpatient care & Ref. & & & & \multirow{3}{*}{0.51} \\
\hline Outpatient care & 0.97 & 0.37 & 2.52 & 0.95 & \\
\hline Day hospital & 0.56 & 0.20 & 1.52 & 0.26 & \\
\hline \multicolumn{6}{|l|}{ Where did you receive the diagnosis? } \\
\hline In my hospital room & Ref. & & & & \multirow{4}{*}{0.46} \\
\hline In a private office & 1.17 & 0.58 & 2.36 & 0.67 & \\
\hline In a public space & 1.26 & 0.25 & 6.34 & 0.78 & \\
\hline Other (by phone or discharge letter) & 0.34 & 0.08 & 1.51 & 0.16 & \\
\hline \multicolumn{6}{|c|}{ Were other people present at the time of the diagnosis? } \\
\hline No & Ref. & & & & \multirow{3}{*}{0.50} \\
\hline My family members & 1.35 & 0.62 & 2.95 & 0.45 & \\
\hline Other people & 0.76 & 0.23 & 2.56 & 0.66 & \\
\hline \multicolumn{6}{|c|}{ How long did the communication of the diagnosis take? } \\
\hline Less than 15 minutes & Ref. & & & & \multirow{4}{*}{$<0.0001$} \\
\hline 15-30 minutes & 2.71 & 1.33 & 5.51 & $<0.01$ & \\
\hline $30-60$ minutes & 8.63 & 2.74 & 27.23 & $<0.01$ & \\
\hline More than 60 minutes & 1.94 & 0.20 & 18.73 & 0.57 & \\
\hline \multicolumn{6}{|c|}{ Did the neurologist provide you with the adequate emotional support? } \\
\hline No & Ref. & & & & \multirow{3}{*}{$<0.0001$} \\
\hline A little bit & 7.78 & 3.18 & 19.04 & $<0.0001$ & \\
\hline Yes & 31.29 & 10.74 & 91.19 & $<0.0001$ & \\
\hline \multicolumn{6}{|c|}{ Did the neurologist tailor the communication to your profile? } \\
\hline No & Ref. & & & & \multirow{3}{*}{$<0.0001$} \\
\hline A little bit & 4.53 & 2.05 & 10.01 & $<0.001$ & \\
\hline Yes & 10.44 & 3.76 & 29.0 & $<0.0001$ & \\
\hline
\end{tabular}


how close to the onset of symptoms our survey had been administered, and it may prevent drawing definitive conclusions from our study.

Anyway, our study should be considered a pilot experience aimed, first of all, at sensitizing people to this issue and analyzing both the awareness of one's limitations and need for improvement. A thorough analysis of both patient's and neurologist' perspectives on this issue may represent the best option to plan further improvements.

An important extension of the current work would be to prospectively evaluate evolving ways and strategies of disclosing the diagnosis of MS over time (i.e., the impact of new health related technologies) and providing information about the disease that meet patients preferences and expectations.

Such efforts are important, as providing additional individualized news may effectively improve acceptance of MS diagnosis, which may influence not only the adherence to a chronic DMT but also the patients' quality of life.

\section{Disclosure}

Vittorio Martinelli has received personal compensation for activities with Biogen Dompe, Merck Serono, Bayer Schering, Teva, and Sanofi Aventis as a speaker. Giancarlo Comi has received compensation for consulting services and/or speaking activities from Bayer Schering Pharma AG, Serono Symposia International Foundation, Merck Serono International, Teva, Sanofi Aventis, and Biogen Dompe.

\section{Conflict of Interests}

The authors declare that there is no conflict of interests regarding the publication of this paper.

\section{References}

[1] L. Dennison, R. Moss-Morris, and T. Chalder, "A review of psychological correlates of adjustment in patients with multiple sclerosis," Clinical Psychology Review, vol. 29, no. 2, pp. 141-153, 2009.

[2] A. Giordano, F. Granella, A. Lugaresi et al., "Anxiety and depression in multiple sclerosis patients around diagnosis," Journal of the Neurological Sciences, vol. 307, no. 1-2, pp. 86-91, 2011.

[3] C. H. Polman, S. C. Reingold, B. Banwell et al., "Diagnostic criteria for multiple sclerosis: 2010 revisions to the McDonald criteria," Annals of Neurology, vol. 69, no. 2, pp. 292-302, 2011.

[4] G. Comi, M. Filippi, F. Barkhof et al., "Effect of early interferon treatment on conversion to definite multiple sclerosis: a randomised study," The Lancet, vol. 357, no. 9268, pp. 1576-1582, 2001.

[5] A. C. J. W. Janssens, J. B. D. Boer, N. F. Kalkers, J. Passchier, P. A. V. Doorn, and R. Q. Hintzen, "Patients with multiple sclerosis prefer early diagnosis," European Journal of Neurology, vol. 11, no. 5, pp. 335-337, 2004.

[6] S. Kern, W. Schrempf, H. Schneider, T. Schultheiß, H. Reichmann, and T. Ziemssen, "Neurological disability, psychological distress, and health-related quality of life in MS patients within the first three years after diagnosis," Multiple Sclerosis, vol. 15, no. 6, pp. 752-758, 2009.
[7] Y. Suh, R. W. Motl, and D. C. Mohr, "Physical activity, disability, and mood in the early stage of multiple sclerosis," Disability and Health Journal, vol. 3, no. 2, pp. 93-98, 2010.

[8] P. Arnett and J. J. Randolph, "Longitudinal course of depression symptoms in multiple sclerosis," Journal of Neurology, Neurosurgery and Psychiatry, vol. 77, no. 5, pp. 606-610, 2006.

[9] M. Elian and G. Dean, "To tell or not to tell the diagnosis of multiple sclerosis," The Lancet, vol. 326, no. 8445, pp. 27-28, 1985.

[10] L. Fallowfield and V. Jenkins, "Communicating sad, bad, and difficult news in medicine," The Lancet, vol. 363, no. 9405, pp. 312-319, 2004.

[11] A. C. J. W. Janssens, D. Buljevac, P. A. van Doorn et al., "Prediction of anxiety and distress following diagnosis of multiple sclerosis: a two-year longitudinal study," Multiple Sclerosis, vol. 12, no. 6, pp. 794-801, 2006.

[12] E. M. Haberfellner and H. Markut, "Patient education in multiple sclerosis: experiences and wishes of affected patients," Wiener Medizinische Wochenschrift, vol. 145, no. 9, pp. 218-222, 1995.

[13] P. G. Papathanasopoulos, A. Nikolakopoulou, and N. J. Scolding, "Disclosing the diagnosis of multiple sclerosis," Journal of Neurology, vol. 252, no. 11, pp. 1307-1309, 2005.

[14] P. Papathanasopoulos, L. Messinis, E. Lyros, A. Nikolakopoulou, E. Gourzoulidou, and S. Malefaki, "Communicating the diagnosis of multiple sclerosis. Results of a survey among Greek neurologists," Journal of Neurology, vol. 255, no. 12, pp. 1963$1969,2008$.

[15] R. F. Brown, E. M. Valpiani, C. C. Tennant et al., "Longitudinal assessment of anxiety, depression, and fatigue in people with multiple sclerosis," Psychology and Psychotherapy: Theory, Research and Practice, vol. 82, no. 1, pp. 41-56, 2009.

[16] V. Martinelli, A. Ghezzi, E. Montanari, M. Radaelli, G. Comi, and R. Bossa, "Disclosing the diagnosis of multiple sclerosis: the Profile Project," Journal of Neurology, vol. 259, no. 12, pp. 26052610, 2012.

[17] C. Heesen, J. Kolbeck, S. M. Gold, H. Schulz, and K. H. Schulz, "Delivering the diagnosis of MS-results of a survey among patients and neurologists," Acta Neurologica Scandinavica, vol. 107, no. 5, pp. 363-368, 2003.

[18] A. Solani, N. Aquarone, E. Pucci et al., "Communicating the diagnosis of a multiple sclerosis-a qualitative study," Multiple Sclerosis, vol. 13, no. 6, pp. 763-769, 2007.

[19] A. Solari, V. Martinelli, M. Trojano et al., "An information aid for newly diagnosed multiple sclerosis patients improves disease knowledge and satisfaction with care," Multiple Sclerosis, vol. 16, no. 11, pp. 1393-1405, 2010. 


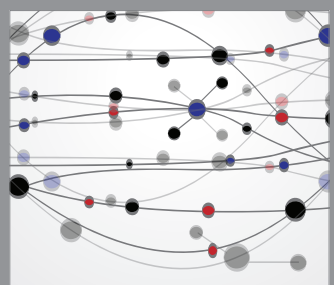

The Scientific World Journal
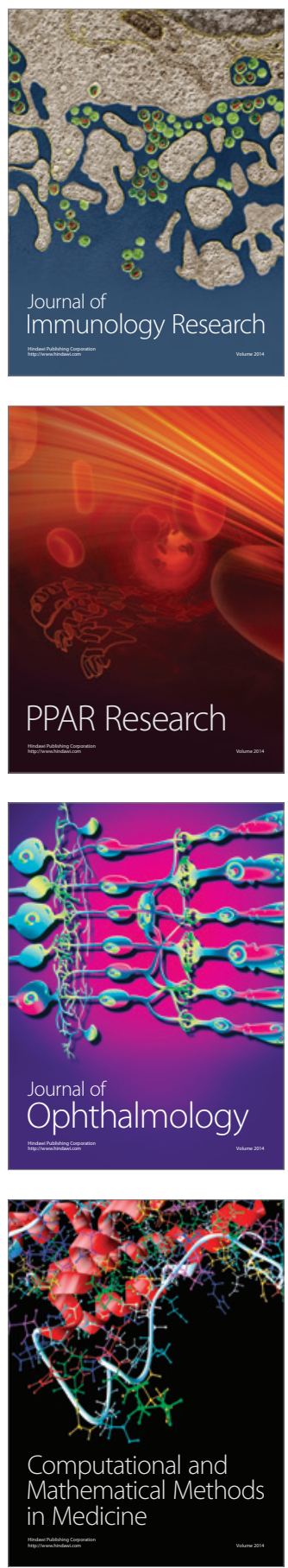

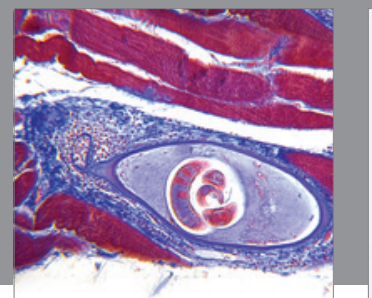

Gastroenterology

Research and Practice
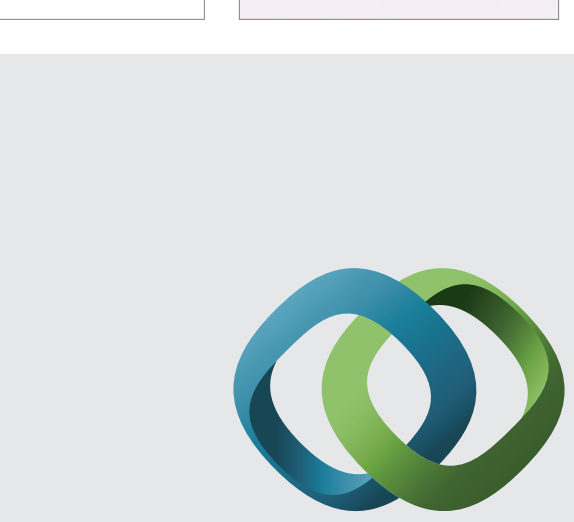

\section{Hindawi}

Submit your manuscripts at

http://www.hindawi.com
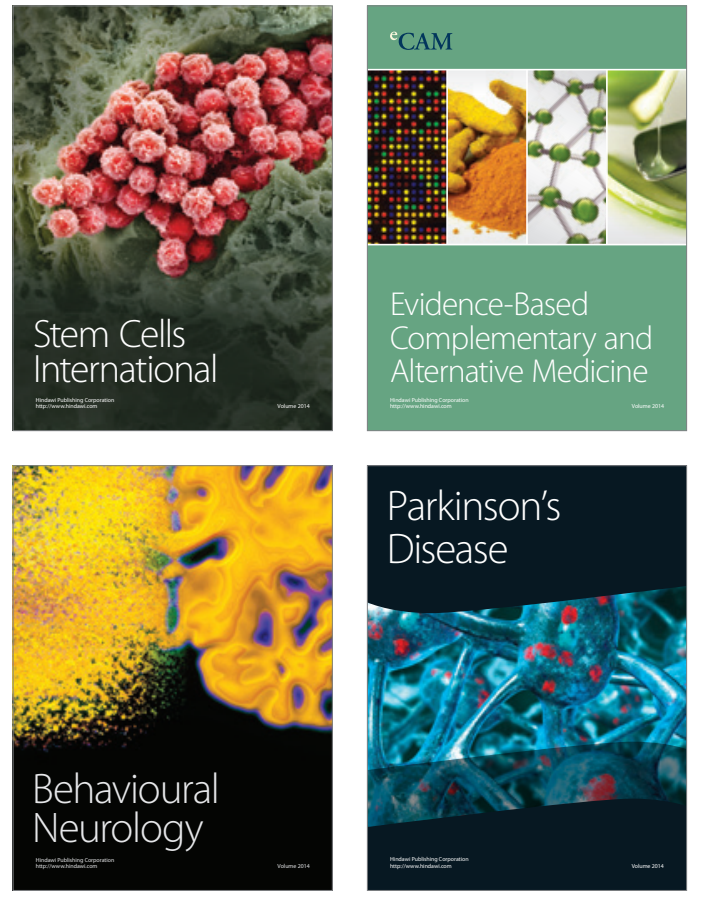
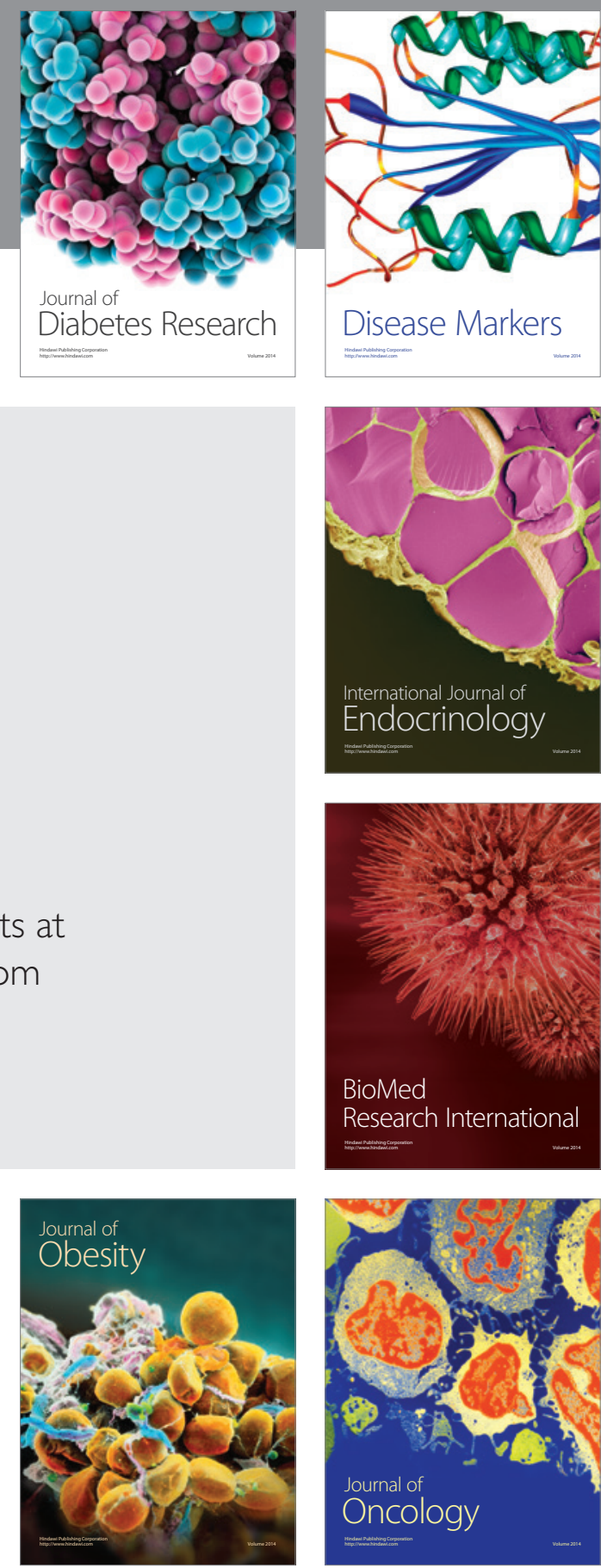

Disease Markers
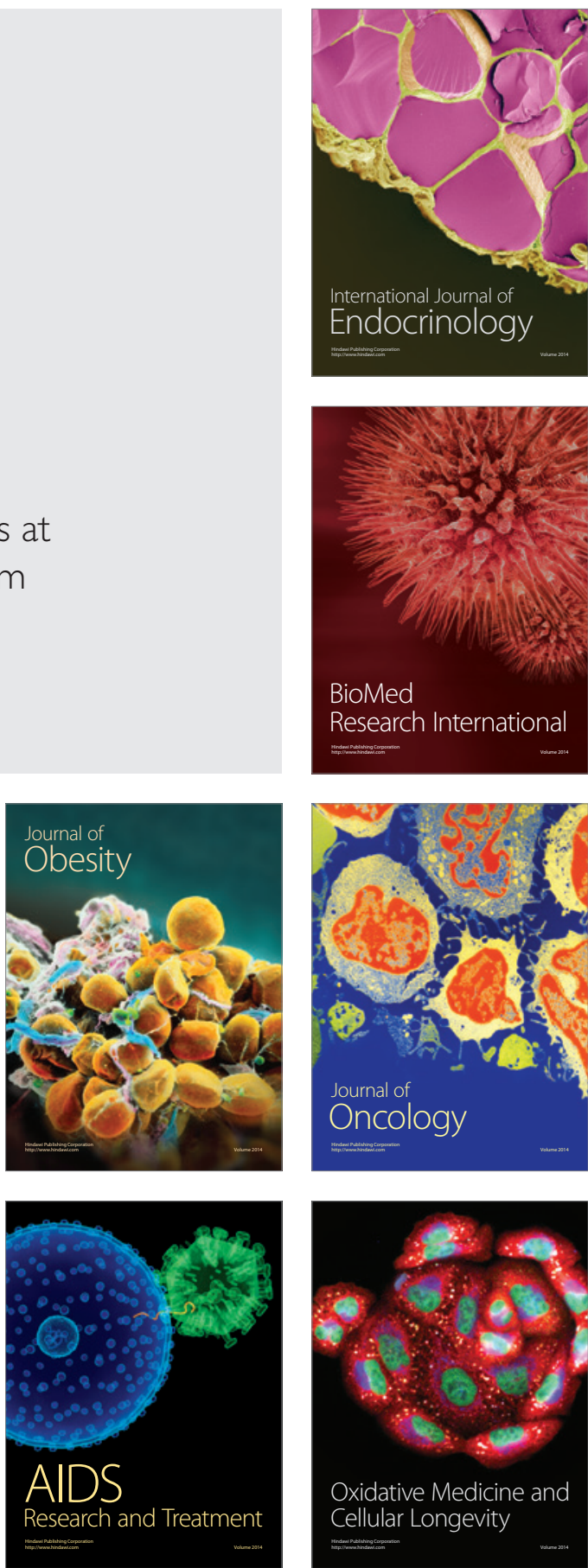